\title{
BIBLIOGRAPHY
}

1. C. Chevalley, Theory of Lie groups, Princeton University Press, 1946.

2. D. Montgomery and L. Zippin, Topological transformation groups, Interscience Press, 1955.

3. H. Seifert, Topologie dreidimensionaler gefaserter Räume, Acta Math. vol. 60 (1932) pp. 147-238.

4. G. T. Whyburn, Analytic topology, Amer. Math. Soc. Colloquium Publications, vol. $28,1942$.

RUTGERS UNIVERSITY

\section{A NOTE ON RECURRENT FLOWS}

CHARLES DAVID GORMAN

Let $R$ be a metric space and let $f: R \times\{t \mid t$ is real $\} \rightarrow R$ be a continuous flow in $R$. The flow $f$ is said to be recurrent (in the sense of Gottschalk and Hedlund) provided that if $\epsilon$ and $s$ are positive real numbers, then there exists a real number $t$ such that $t>s$ and $\rho(x, f(x, t))$ $<\epsilon$ for all $x \in R$.

THEOREM. Consider the statements:

(I) $f$ is recurrent,

(II) There exists a sequence $t_{1}<t_{2}<\cdots \rightarrow+\infty$ of real numbers such that $\lim _{n \rightarrow \infty} f\left(A, t_{n}\right)=A$ for every closed subset $A$ of $R$,

(III) There exists a sequence $t_{1}<t_{2}<\cdots \rightarrow+\infty$ of real numbers such that $\lim \sup _{n \rightarrow \infty} f\left(A, t_{n}\right) \subset A$ for every closed subset $A$ of $R$.

Then,

(1) I implies II; II implies III,

(2) If $R$ is compact, then I, II, III are equivalent.

Proof. Assume I. There exists a sequence $t_{1}<t_{2}<\cdots \rightarrow+\infty$ of real numbers such that $\rho\left(x, f\left(x, t_{n}\right)\right)<1 / n$ for all $x \in R$ and all positive integers $n$. Let $A$ be a closed subset of $R$. If $x \in A$, then $f\left(x, t_{n}\right) \rightarrow x$. Hence $A \subset \lim \inf _{n \rightarrow \infty} f\left(A, t_{n}\right)$. Suppose $x \in \lim \sup _{n \rightarrow \infty} f\left(A, t_{n}\right)$. There exist $x_{1}, x_{2}, \cdots \in A$ and a sequence $n_{1}<n_{2}<\cdots \rightarrow+\infty$ of positive integers such that $\rho\left(x, f\left(x_{i}, t_{n_{i}}\right)\right) \rightarrow 0$. Since $\rho\left(x_{i}, f\left(x_{i}, t_{n_{i}}\right)\right)<1 / n_{i}$ for all positive integers $i$, it follows that $\rho\left(x_{i}, f\left(x_{i}, t_{n_{i}}\right)\right) \rightarrow 0$ whence $x_{i} \rightarrow x$ and $x \in A$. Hence $\lim \sup _{n \rightarrow \infty} f\left(A, t_{n}\right) \subset A$. This proves that I implies II.

Presented to the Society, September 2, 1955 ; received by the editors January 5 , 1955 and, in revised form, on April 18, 1955. 
It is immediate that II implies III.

Assume III. Let $R$ be compact. Let $\epsilon$ and $s$ be positive real numbers. There exists a finite sequence $A_{1}, \cdots, A_{k}$ of closed subsets of $R$ such that $A_{1} \cup \cdots \cup A_{k}=R$ and $\operatorname{diam}\left(A_{i}\right)<\epsilon / 2(i=1, \cdots, k)$. Choose a positive integer $n$ so that $t_{n}>s$ and $f\left(A_{i}, t_{n}\right) \subset N\left(A_{i}, \epsilon / 2\right)$ $(i=1, \cdots, k)$ where $N(A, \delta)$ denotes the $\delta$-neighborhood of the set $A$. Let $x \in R$. Then $x \in A_{j}$ for some $j(j=1, \cdots, k)$. It follows that $\rho\left(f\left(x, t_{n}\right), y\right)<\epsilon / 2$ for some $y \in A_{j}$. Since $x, y \in A_{j}$ we have $\rho(x, y)$ $<\epsilon / 2$. Hence, $\rho\left(x, f\left(x, t_{n}\right)\right)<\epsilon$. This proves (2).

We remark that the above theorem and proof apply to discrete flows. Also the theorem answers a question by Nemyckii concerning a characterization of pseudo-minimal sets (cf. V. V. Nemyckii, Topological problems of the theory of dynamical systems, American Mathematical Society Translation, no. 103, p. 69).

The author wishes to acknowledge the assistance provided by the referee in improving the statement of the theorem and in shortening the proof.

UNIVERSITY OF MISSOURI 